DOI: http://dx.doi.org/10.18569/tempus.v13i2.2682

\title{
Responsabilidade da população, objectivo da cooperação na região da saúde da Região de Lausanne (Suíça)? um estudo de caso.
}

\section{La responsabilité populationnelle, objectif de la coopération au sein du Réseau Santé Région Lausanne (Suisse)? une étude de cas.}

\section{Population responsibility, objective of cooperation within the health network of Lausanne Region (Switzerland)? a case study.}

Philippe Anhorn ${ }^{1}$

RESUMO: A Rede Regional de Saúde de Lausanne (RSRL) é a maior (em termos de população) das quatro redes de saúde que cobrem o território do Distrito de Vaud, na Suíça. As qualidades destas redes (projectos inovadores, contribuição para a fluidez do sistema de saúde, etc.) foram frequentemente elogiadas. Contudo, desde a sua criação, no final da década de 1990, a sua eficiência tem sido difícil de demonstrar. Para tal, os seus membros - prestadores de cuidados hospitalares e ambulatórios - deveriam ter aumentado significativamente o seu nível de cooperação, por exemplo, através da partilha de um número crescente de actividades e projectos, reunindo simultaneamente os recursos necessários para a sua execução. Após o fracasso, em 2012, da introdução do managedcare sob o controle das seguradoras no âmbito federal e, em 2017, de um projeto de lei para criar quatro entidades regionais de saúde comunitária sob o controle do Estado com responsabilidade populacional, abre-se uma janela de oportunidade para que os profissionais de saúde agrupados dentro das redes decidam por si mesmos, ou seja, numa abordagem bottomup, intensificar gradualmente a sua cooperação em torno de projectos inovadores e significativos, até seepossivel reivindicar esta responsabilidade colectiva, pelo menos para a parte da população mais afectada pelos seus serviços: os idosos e as pessoas em perda de autonomia. O Projecto de Cuidados Anticipados (PCA), que se encontra numa fase piloto no âmbito da RSRL com a participação de outras redes e autoridades distritais, poderá ser um primeiro passo nesta direcção.

1 Réseau Santé Région Lausanne (RSRL), Suisse. Email : Philippe.Anhorn@,rsrl.ch 
Palavras-chave: Política. Planeamento e gestão dos serviços de saúde. Coordenação e acompanhamento dos cuidados de saúde. Financiamento dos cuidados de saúde. Financiamento de base populacional.

ABSTRACT: Réseau Santé Région Lausanne (RSRL) is the most important (in terms of population) of four healthcare networks covering the Canton of Vaud in Switzerland. The qualities of these networks (innovative projects, contribution to the fluidity of the health system, etc.) have often been praised. But since their creation in the late 1990s, their efficiency has hardly been demonstrated. To do this, their members - both stationary and ambulatory care providers - would have had to significantly increase their level of cooperation, for example by sharing an increasing number of activities and projects while pooling the resources needed for their implementation. After the failure in 2012 of the introduction of managed care under the control of insurers at the federal level and then, in 2017, of a bill to create under the control of the State four regional community health organizations with a population responsibility, an opportunity raises for the care providers grouped within the networks to decide themselves, in a bottom-up approach, to gradually intensify their cooperation around innovative and meaningful projects, to be able to claim this collective responsibility, at least for the part of the population mainly concerned by their services: the elderly and fragile people. The Advanced Care Project (PAS), piloted within the RSRL with the participation of other networks and cantonal State services, could be a first step in this direction.

Keywords: Health Policy. Planning and Management. Health Care Coordination and Monitoring. Healthcare Financing. Capitation Fee.

RÉSUMÉ: Le Réseau Santé Région Lausanne (RSRL) est le plus important (en termes de population) des quatre réseaux de soins couvrant le territoire du Canton de Vaud en Suisse. Les qualités de ces réseaux (projets innovateurs, contribution à la fluidité du système de santé, etc.) ont souvent été saluées. Mais, depuis leur création à la fin des années 1990, leur efficience a été difficilement démontrée. Pour y parvenir, il aurait fallu que leurs membres - prestataires de soins stationnaires et ambulatoires - élèvent significativement leur degré de coopération, par exemple en partageant un nombre croissant d'activités et projets tout en mutualisant les ressources nécessaires à leur mise en œuvre. Après l'échec en 2012 de l'introduction du managed care sous le contrôle des assureurs au plan fédéral puis, en 2017, d'un projet de loi visant à créer sous le contrôle de l'État quatre entités régionales de santé communautaire dotées d'une responsabilité populationnelle, une fenêtre d'opportunité s'ouvre pour que les prestataires de soins regroupés au sein des réseaux décident eux-mêmes, c'est à dire dans une démarche bottom up, d'intensifier graduellement leur coopération autour de projets innovateurs et porteurs de sens, jusqu'à être en mesure de revendiquer cette responsabilité collective, au moins pour la partie de la population principalement concernée par leurs prestations : les personnes âgées et en perte d'autonomie. Le projet anticipé des soins (PAS), en phase pilote au sein du RSRL avec la participation des autres réseaux et des instances cantonales, pourrait être une première étape dans cette direction.

Mots-clés: Politique. Planification et gestion des services de santé. Coordination et surveillance des soins de santé. Financement des soins de santé. Financement à la capitation.

Tempus, actas de saúde colet, Brasília, 13(2), 235-245, jun, 2019.

ISSN 1982-8829 


\section{INTRODUCTION}

Cet article constitue une étude de cas ${ }^{1}$ sur la situation spécifique du Réseau Santé Région Lausanne au début de l'année 2019, confronté à la nécessité d'une performance accrue pour faire face, tant au plan quantitatif que qualitatif, aux enjeux du vieillissement de la population dans les prochaines décennies. Dans les systèmes de santé des pays industrialisés, l'un des leviers de l'amélioration de la performance réside dans l'élévation du degré de coopération entre les acteurs ${ }^{2,3}$. Ces degrés se définissent par différents niveaux qui vont de la simple liaison (linkage) à la coordination jusqu'à la pleine intégration ${ }^{4}$. Cette étude de cas est réalisée sur la base des références bibliographiques mentionnées ainsi que d'une dizaine d'entretiens préliminaires conduits par l'auteur avec les principaux responsables des milieux concernés, en vue du cadrage d'une thèse de doctorat qu'il rédige sur le même sujet.

\section{CONTEXTE}

Le Réseau Santé Région Lausanne (RSRL) est l'un des quatre réseaux de fournisseurs de soins reconnus d'intérêt public actifs sur le territoire du Canton de Vaud. La Loi sur les réseaux de soins de $2007^{5}$ fait obligation à tous les fournisseurs de soins subventionnés d'adhérer et de contribuer au réseau de leur région. Le RSRL regroupe ainsi un hôpital universitaire (CHUV, Lausanne), 4 autres hôpitaux de soins aigus et de réadaptation, 19 centres médico-sociaux (CMS = aide et soins à domicile), 53 établissements médico-sociaux (EMS = maisons de retraite médicalisées), 56 membres associés privés (cliniques, organisations de soins à domicile), plus de 800 médecins et autres professionnels en libre pratique et enfin 63 communes territoriales dont les élus sont considérés comme porte-paroles légitimes des besoins de la population, non seulement malade mais également bien-portante. Le RSRL emploie directement 86 collaborateurs (principalement des infirmiers/-ères de liaison) et dispose d'un budget annuel d'environ 11 millions de francs suisses.

Le RSRL est constitué sous forme d'association de droit privé. Son organe suprême est l'Assemblée générale. Celle-ci élit un Comité directeur constitué de 2 représentants pour chacun des 5 groupes de membres (Hôpitaux de soins aigus et réadaptation, Etablissements médicosociaux, Centre médico-sociaux, Médecins, Communes). Ce Comité peut être assimilé à une table de concertation tactique ${ }^{6}$, lieu propice pour la définition de projets régionaux et leur financement par l'allocation des ressources mises en commun (subventions, cotisations et contributions des membres).

Les réseaux dans le Canton de Vaud ont pour mission d'améliorer la coordination et la continuité des soins, d'orienter les usagers dans le système de santé, de promouvoir la collaboration institutionnelle au niveau régional et la mise en œuvre de programmes cantonaux de santé publique. Leurs prestations s'adressent principalement aux personnes âgées présentant des risques de déclin fonctionnel. 
L'une des prestations principales des réseaux est le Bureau régional d'information et de liaison (BRIO). Celui-ci emploie des infirmiers et infirmières de liaison qui, à l'hôpital comme dans la communauté, interviennent selon un processus clinique comprenant le repérage des signes de fragilité, l'évaluation (ou co-évaluation avec les soignants de $1^{\text {re }}$ ligne), la définition du projet d'orientation, l'orientation du patient et enfin le suivi. A cette fin, le BRIO centralise toutes les annonces de lits disponibles (courts ou longs séjours) dans les EMS de sa région et les alloue en fonction des demandes, selon un système de priorisation qui tient compte des souhaits des usagers, de leurs besoins (adéquation entre les profils de dépendance et les options de soins) et des disponibilités. Cette prestation est financée à $40 \%$ par une subvention de l'État et à $60 \%$ par un prélèvement sur les ressources des membres institutionnels concernés (hôpitaux, EMS, soins à domicile). Le financement du BRIO est calculé « à la capitation », c'est-à-dire que le RSRL reçoit le budget correspondant aux coûts (salaires, charges sociales et overheads) de 2 postes équivalents plein-temps (EPT) par tranche de 1'000 habitants âgés de 75 ans ou plus sur son territoire.

\section{L'INTÉGRATION DANS LES SYSTÈMES DE SANTÉ}

Dans de nombreux pays de l'OCDE, l'organisation du système de santé s'est développée dès la fin des années 1990 dans le sens d'une intégration accrue des services, à des degrés divers ${ }^{7,8}$ et de manières très différentes selon que l'organisateur de l'intégration se trouve être un assureur, un groupe de fournisseurs ou l'État.

La forme la plus développée de soins intégrés sous gouvernance étatique est sans doute celle mise en œuvre au Royaume Uni dans le cadre du NHS ${ }^{9}$. A l'échelle locale, des Primary Care Trusts (PCT), c'est-à-dire littéralement des " groupes de soins primaires », sont constitués de prestataires de premier recours (principalement des médecins généralistes) qui sont chargés d'une mission de santé publique à l'échelle de leur région et dotés de ressources leur permettant de contracter pour l'achat de soins secondaires spécialisés. Les PCT sont principalement financés à la capitation par le ministère britannique de la santé, « en fonction d'une formule nationale qui tient compte des caractéristiques démographiques et socio-économiques de la région et des variations locales du coût du travail et du capital. (...) Les PCT doivent veiller à ce que leurs dépenses annuelles ne dépassent pas l'enveloppe qui leur a été allouée ». En 2016, chaque PCT exerçait une responsabilité populationnelle pour environ 150'000 habitants alors qu'une réorganisation était en cours pour constituer par fusions des régions fortes de 500'000 habitants. Pour de nombreuses raisons qui ne sont pas discutées ici, le système de santé britannique a souvent été critiqué en raison de ses mauvaises performances. Celles-ci ne devraient pas pour autant occulter le caractère très innovant d'une politique de santé déterminée en matière d'intégration des soins.

Aux États-Unis, le modèle de soins intégrés le plus abouti est celui d'un réseau de fournisseurs qui, avec ou sans but lucratif, conditionne l'accès à ses services pour la population d'une région à la souscription d'un plan d'assurance. Un exemple bien connu en Europe d'une telle organisation (grâce à une politique de communication active et de nombreuses collaborations scientifiques) 
est l'Intermountain Healthcare ${ }^{10}$, basé en Utah, qui sert environ $800^{\prime} 000$ assurés avec un réseau associatif sans but lucratif constitué de 22 hôpitaux et plus de 1'500 médecins et fournisseurs de soins divers.

Comme dans tous les domaines de la santé publique, le Québec s'est montré très innovant dans les différents modèles de soins intégrés. On trouve des synthèses très complètes des différentes expériences menées ${ }^{11,12}$.

En France, parmi 54 projets d'innovation organisationnelle rendus possibles et financés à hauteur de 30 millions d'euros par le nouvel article 51 de la Loi de financement de la sécurité sociale $^{2}$, la Fédération hospitalière de France (FHF) lance un projet d'intégration clinique basé sur la responsabilité populationnelle dans l'Aube et le Sezennais, la Cornouaille, les Deux-Sèvres et la Haute-Saône. Lors de la présentation du plan « Ma santé 2022 » le 18 septembre 2018, Emmanuel Macron lui-même a indiqué vouloir encore « amplifier » ces développements. L'un des six rapports présentés à cette occasion, intitulé « Repenser l'organisation territoriale des soins ${ }^{13}$ développe le concept, les enjeux et les objectifs de la responsabilité populationnelle ou territoriale.

En Suisse, il existe un mouvement pour la constitution de « groupes de fournisseurs », souvent autour d'un cabinet au sein duquel plusieurs médecins sont associés et qui s'adjoignent des soins infirmiers, éventuellement une organisation de soins à domicile (OSAD), de la physiothérapie, radiologie, etc. C'est par exemple le cas de Salutomed $\mathrm{AG}^{14}$, une petite organisation active dans le Canton de Berne. Certaines de ces organisations ont pour centre de gravité un hôpital, comme par exemple le groupe de l'Ensemble hospitalier de la Côte $(\mathrm{EHC})^{15}$ dans le Canton de Vaud qui regroupe dans une association sans but lucratif 3 hôpitaux de soins aigus ou de réadaptation, 2 EMS et 2 cabinets de groupe. Dans le Canton de Genève (et, par « débordement », dans d'autres cantons de Suisse romande, le réseau Delta ${ }^{16}$ est également constitué principalement par des médecins de 1 er recours acquis à la philosophie du management par la qualité ${ }^{17}$. Créé en 1992, Delta regroupe aujourd'hui plus de 600 médecins et assure la santé de plus de 250000 patients. Grâce au contrat Médecin de famille ou HMO des assurances partenaires de Delta, les patients peuvent économiser de 10 à 20\% sur leur prime d'assurance maladie obligatoire. Également sans but lucratif, le Réseau Delta investit ses bénéfices dans des projets novateurs, notamment en gériatrie avec la création à Onex d'une véritable Maison de médecine de premier recours, appelée Cité Générations ${ }^{18}$ qui regroupe des cabinets médicaux, des soins infirmiers et des lits d'observation principalement destinés à une patientèle âgée et fragile. Face à une organisation du système de santé qui, en Suisse, reste hyper-fragmentée, les co-fondateurs du Réseau Delta, les Dr Philippe Schaller et Marc-André Raetzo qui en ont pourtant (ou justement) exploité toutes les possibilités, plaident depuis longtemps pour une évolution du cadre réglementaire et financier en Suisse qui permettrait d'aller vers des modèles d'intégration beaucoup plus poussés ${ }^{19}$.

Cet état des lieux n'est évidemment pas exhaustif mais mentionne les principaux éléments qui 
fondent notre réflexion

\section{EVOLUTION PRÉVISIBLE}

Dans notre région, comme partout dans les pays industrialisés, la population vieillit et les maladies chroniques et dégénératives se multiplient. La pression sur les services de santé dans le Canton de Vaud sera multipliée par 2, voire par 3 à l'horizon $2040^{20}$ Dans le même temps, il est avéré que les ressources (financières et humaines) ne pourront augmenter linéairement par rapport à cette croissance des besoins. D'autres facteurs contribuent à la croissance continue des coûts de la santé : progrès technologique, hyper-spécialisation, prix des médicaments, etc. La Suisse dispose aujourd'hui de l'un des meilleurs systèmes de santé au monde, mais aussi du plus cher à pouvoir d'achat égal parmi les pays de l'OCDE ${ }^{21}$. C'est aussi l'un des pays où la part directement à charge des patients (out of pocket), par le biais des franchises, quotes-parts et participations, est la plus élevée : environ $30 \%$, soit le double de la part moyenne dans les pays qui nous entourent. Le coût des primes d'assurance-maladie pour les ménages est l'un des objets de préoccupation les plus importants de la population. Il pourrait poser à relativement court terme des problèmes de cohésion sociale. De nombreuses réflexions sont en cours pour maîtriser les coûts de la santé, à plusieurs échelons : celui de la Confédération ${ }^{22}$, dans certains des 26 cantons souverains en matière d'organisation de leur système de santé23 et au niveau des associations faîtières ou des ordres professionnels.

La coordination des soins est dûment reconnue par la Confédération comme une piste de maîtrise des coûts de la santé. C'est l'un des axes de la stratégie « Santé $2020 »^{24} \mathrm{du}$ Conseil fédéral. Pourtant, hormis certains acteurs privés qui opèrent de manière opportuniste par voie de fusions/acquisitions, il n'existe pas de projet de service public qui porte sur la reconfiguration des systèmes de santé sous l'angle d'une véritable intégration des services ${ }^{25}$, normative, structurelle, budgétaire, fonctionnelle, clinique, etc. ${ }^{26}$. Le concept de managed care a été rejeté par $76 \%$ de la population lors d'un référendum en $2012^{27}$. Le projet d'alors donnait aux assureurs la haute main sur le fonctionnement du système, notamment en supprimant leur obligation de contracter avec les fournisseurs de soins. Les citoyens ont principalement redouté la perte du libre choix de leur médecin. Cet épisode complique la reprise à l'échelon fédéral d'une réflexion sur le managed care, même avec un modèle qui ne serait pas piloté par les assureurs mais plutôt par les pouvoirs publics et/ou par les prestataires de soins.

Indépendamment de la question des coûts, la capacité du système de santé à absorber la vague démographique qui s'annonce, et à mobiliser les ressources nécessaires (financières et humaines) pour répondre aux besoins, est principalement de la responsabilité des cantons. Ceux-ci sont en effet souverains en matière d'organisation de leurs services de santé, la Confédération ne jouant qu'un rôle subsidiaire, régulateur au travers de la loi sur l'assurance maladie (LAMal), et de haute surveillance sur quelques thématiques spécifiques comme les maladies infectieuses. Si le Canton de Vaud a été pionnier dans la création de réseaux de soins coordonnés dès la fin des années 
1990, ceux-ci sont restés centrés sur des tâches très spécifiques (cf. BRIO ci-dessus) ou sur des projets d'envergure modeste, avec des enjeux financiers réduits et principalement fondés sur le bon vouloir de leurs membres. Leurs résultats qualitatifs (projets innovateurs, contribution à la fluidité du système, etc.) ont souvent été salués, mais leur efficience peine à être démontrée. Pour y parvenir, il faudrait que leurs membres renoncent à une part significative de leurs ressources propres pour les mettre au « pot commun » du réseau, qu'ils s'entendent sur leur redistribution en fonction de critères fixés collectivement, et que leurs performances en découlant soient comparées à celles d'acteurs similaires distribuant les mêmes prestations mais sans dispositif spécifique de coordination. Ceci est compliqué tant que chaque groupe d'acteurs est régi par une réglementation distincte et financé principalement à l'activité (à l'acte pour le secteur ambulatoire et au forfait pour le secteur stationnaire). Chaque institution ou professionnel exerce donc une responsabilité distincte sur sa gestion. Malgré les évidentes incitations négatives qui en découlent (multiplication des actes par les prestataires, au mieux par ignorance ou défaut de coordination, au pire pour maximiser leurs revenus), la question d'une responsabilisation collective des acteurs du système de santé, qui avait été évoquée mais non retenue à la fin des années 1990 lors de la création de réseaux de soins, ne s'est jamais sérieusement posée à nouveau dans le Canton de Vaud jusqu'en 2017.

\section{VERS UNE RESPONSABILISATION POPULATIONNELLE?}

Au printemps 2017, l'État de Vaud a mis en consultation un avant-projet de $1 \mathrm{i}^{28}$.proposant une nouvelle organisation des services de santé en 4 " régions de santé » exerçant chacune une responsabilité populationnelle. Ces régions auraient bénéficié d'un financement mixte incluant une part de rémunération (comme aujourd'hui) à l'activité, une part de capitation en fonction du nombre d'habitants par région pondéré par l'état de santé de la population (selon une stratification découlant du système d'évaluation multidimensionnelle standardisée interRAIC qui doit être prochainement introduit), et enfin une part à la performance (selon des critères à définir). Cependant, le projet mis en consultation a été perçu à la fois comme trop contraignant sur les aspects structurels et administratifs, et trop vague sur les modalités de reconfiguration des systèmes de santé régionaux et sur la capacité des acteurs à exercer cette fameuse responsabilité populationnelle. Les milieux consultés se sont prononcés majoritairement contre le projet, arguant que celui-ci avait été élaboré de manière trop peu participative, ne contenait pas d'analyse suffisamment approfondie du fonctionnement existant, ni de scénario détaillé pour les changements proposés. Le Conseil d'Etat (gouvernement cantonal) a suspendu le projet ${ }^{29}$, avançant que l'opposition était avant tout idéologique (le ministre de la santé étant socialiste tandis que la plupart des milieux concernés sont plutôt libéraux).

Peu après ce retrait, en automne 2017, le Département vaudois de la santé et de l'action sociale a adressé aux quatre réseaux de soins du Canton un cahier des charges visant à décliner l'un des processus non contestés de l'avant-projet, celui de la « Réponse à l'urgence ». Il s'agit notamment d'éviter les hospitalisations inappropriées en renforçant les ressources communautaires (aide et soins à domicile, garde des médecins, équipes mobiles, structures de jour et de nuit, etc.) Hélas, 
le déploiement de ce dispositif sur le territoire du RSRL n'a pas été confié par le Département cantonal de la santé au RSRL, mais à la Policlinique médicale universitaire (PMU - depuis le $1^{\text {er }}$ janvier 2019 Unisanté : Centre universitaire de médecine générale et santé publique) de Lausanne. C'est une occasion manquée pour le réseau de tester l'efficience d'une gouvernance renforcée et l'allocation de ressources mutualisées pour un panier de prestations concourant à l'objectif commun d'offrir à la population un dispositif de réponse à l'urgence dans la communauté.

L'allocation des ressources mises en commun semble pourtant être la voie prometteuse pour encourager les acteurs à intensifier leur coopération, pour accroître leur efficience et donc leur productivité dans un contexte de raréfaction des ressources face à la croissance des besoins. Cependant, l'échec du projet de loi susmentionné lors de la consultation tend à montrer qu'on ne peut l'envisager exclusivement sous l'angle financier et réglementaire, sans provoquer le repli des membres des réseaux sur leurs « territoires » (institutionnels, professionnels, philosophiques, politiques, etc.). Dans un environnement globalement toujours plus complexe, la coopération est souvent perçue comme un facteur de complexité supplémentaire. Une perception souvent aggravée par le fait que cette complexité fait émerger, selon les besoins, différentes instances de coordination à différents niveaux, au point qu'on se demande parfois « mais qui coordonne les coordinations? » Si l'on attend des réseaux de soins qu'ils démontrent leur potentiel en matière de réduction (ou tout au moins de maîtrise) des coûts de la santé, il faut les légitimer comme les instances exclusives (et expertes) de la coordination du système de santé régional.

La responsabilité populationnelle, ou responsabilisation collective des acteurs du système de santé à l'échelle de la population de leur région, ne pourra se mettre en place sur la seule base d'une régulation légale, contractuelle ou financière. C'est plutôt par l'identification de valeurs communes (innovation, transparence, participation, solidarité, éthique, etc.) et au travers de la mise en œuvre de projets porteurs de ces valeurs que ces acteurs pourront définir les objectifs communs qu'ils chercheront à atteindre grâce à une responsabilité populationnelle non pas imposée top-down par les assureurs ou les pouvoirs publics, mais revendiquée bottom-up par eux-mêmes, ensemble. Le Réseau Santé Région Lausanne est à un tournant de son évolution. Son développement en tant qu'instance exerçant une responsabilité populationnelle de santé publique à l'échelle de son territoire pourrait se construire de manière progressive et sélective, en s'appuyant sur la mise en œuvre de projets qui portent fortement les valeurs précitées. Un tel projet a vu le jour en 2017 à l'initiative des membres du RSRL et pourrait être l'amorce d'une telle évolution. Il s'agit du Projet Anticipé des Soins ${ }^{30}(\mathrm{PAS})$. Ce projet fait déjà l'objet, à titre pilote, d'un financement conjoint par les acteurs régionaux et l'autorité cantonale. Après un état des lieux éclairant la connaissance et les pratiques respectives sur les questions liées à l'anticipation, à l'autodétermination et au partenariat entre patients et équipes soignantes, les membres du RSRL sont aujourd'hui impliqués dans une phase de co-construction des processus et des outils du projet. Une phase de test aura lieu cette année dans plusieurs milieux de soins au sein du réseau. Cette phase sera accompagnée au plan académique par la Chaire de soins palliatifs gériatriques ${ }^{31}$ du CHUV pour les questions relevant de 
la clinique et de la formation, et par l'auteur de cet article dans le cadre de sa thèse sur les aspects relevant du management, en particulier pour étudier les liens entre un tel projet d'innovation (clinique, organisationnelle, sociale, technologique, etc.), le degré de coopération qu'il nécessite de la part des acteurs concernés et la performance collective qu'il devrait contribuer à améliorer.

\section{RÉFÉRENCES}

1. Yin R-K (1984), « Case study research : design and methods » (Rev. ed), Sage Publications, Beverly Hills, Calif

2. Hirtzlin I (1999), « La coopération entre organisations comme indicateur de la performance publique : exemple du secteur de la santé », Politiques et Management Public, 10727.

3. Hofmarcher M, Oxley H, Rusticelli E (2007), « Improved Health System Performance through better Care Coordination », Documents de travail de l'OCDE sur la santé, $n^{\circ} 30$, Éditions OCDE, Paris

4. Leutz W (1999), «Five Laws for Integrating Medical and Social Services: Lessons from the United States and the United Kingdom », The Milbank Quarterly, 77: 77-110

5. Grand Conseil du Canton de Vaud (2007) [En ligne], « Loi sur les réseaux de soins (LRS) ». 810.02 [consulté le 27 mai 2019]https://www.reseau-sante-region-lausanne.ch/system/ files/2014/02/1oi_sur_les_reseaux_2007.pdf

6. Contandriopoulos A-P, Suárez-Herrera JC, Cupertino de Barros FP, Hartz ZM de A, éds (2018), «Améliorer la performance des systèmes de santé : Concepts, méthodes, pratiques », Montréal: Presses de l’Université de Montréal

7. Contandriopoulos, A-P et al (2001), «Intégration des soins : Dimension et mise en œuvre », Ruptures, revue transdisciplinaire en santé, vol.8, n², pp 38-52.

8. Leutz W (2005), «Reflections on Integrating Medical and Social Care: Five Laws Revisited », Journal of Integrated Care, vol. 13, no 5, pp 3-12.

9. Mason A, et Smith P (2006), «Le système de santé anglais : régulation et rapports entre les différents acteurs », Revue française des affaires sociales, no. 2, pp. 265-284.

10. Ministère des Solidarités et de la Santé [En ligne]. Paris (France): « Expérimenter pour mieux soigner » [modifié le 7 fév 2019 ; cité le 27 mai 2019];https://solidarites-sante.gouv.fr/ systeme-de-sante-et-medico-social/parcours-des-patients-et-des-usagers/article-51-10918/article51

11. Poirier L et al. (2014), « Synthèse des connaissances sur les conditions de mise en œuvre 
des réseaux de service intégrés aux personnes âgées », Institut national de santé publique du Québec

12. Roy DA et al. (2010), « Des réseaux responsables de leur population. Moderniser la gestion et la gouvernance en santé », Ed Le Point en administration de la santé et des services sociaux, Québec

13. Pribile P, Nabet N (2018). [En ligne]. Paris (France). «Repenser l'organisation territoriale des soins » [modifié le 15 oct 2018 ; cité le 27 mai 2019]; https://solidarites-sante.gouv.fr/IMG/ pdf/masante2022_rapport_organisation_territoriale.pdf

14. Salutomed (2019) [En ligne]. Zollikofen (Suisse). « Wir behandeln Menschen verantwortungsvoll » [consulté le 27 mai 2019] https:/www.salutomed.ch/

15. Ensemble Hospitalier de la Côte [En ligne]. Morges (Suisse). [Consulté le 27 mai 2019] http://www.ehc-vd.ch/

16. Réseau Delta [En ligne]. Onex (Suisse). [Consulté le 27 mai 2019] https://reseau-delta.ch

17. Edmunds M, Frank R, Hogan M. (1997), « Managing Managed Care: Quality Improvement in Behavioral Health », Washington (DC): National Academies Press (US)

18. Cité générations [En ligne]. Onex (Suisse). [Consulté le 27 mai 2019] http://www.citegenerations.ch/

19. Schaller P, Gaspoz JM (2008), « Continuité, coordination, intégration des soins : entre théorie et pratique ». Rev Med Suisse 4 : 2034-9

20. Statistique Vaud No 3 (2017) [En ligne]. Lausanne (Suisse): « Prospectif Senior » [consulté le 27 mai 2019] http://www.scris.vd.ch//Data_Dir/ElementsDir/8664/1/F/03_ Prospectif_Seniors.pdf

21. OECD [En ligne] « Ressources pour la santé - Dépenses de santé - OCDE Data » [consulté le 27 mai 2019] http://data.oecd.org/fr/healthres/depenses-de-sante.htm.

22. Confédération Suisse (2017) [En ligne], Berne (Suisse): « Mesures visant à freiner la hausse des coûts dans l'assurance obligatoire des soins / Rapport du groupe d'experts » [consulté le 27 mai 2019] https://www.bag.admin.ch/dam/bag/fr/dokumente/cc/Expertenbericht. pdf.download.pdf

23. Dutoit L, Füglister-Dousse S \& Pellegrini S (2016), « Soins de longue durée dans les cantons : un même défi, différentes solutions. Evolutions 2006-2013 », Obsan Rapport 69, Neuchâtel, Observatoire suisse de la santé. 
24. Confédération Suisse (2017) [En ligne]. « Santé 2020 » [consulté le 27 mai 2019] https:// www.bag.admin.ch/bag/fr/home/strategie-und-politik/gesundheit-2020.html

25. Couturier Y, Belzile L, Bonin L (2016), « L'intégration des services en santé / Une approche populationnelle », Les Presses de l'Université de Montréal. Paramètres

26. Couturier Y, Belzile L et Gagnon D (2011), « Principes méthodologiques de L'implantation du modèle PRISMA portant sur L'intégration des services pour les personnes âgées en perte dáutonomie », Management \& Avenir, vol. 47, no. 7, 2011, pp. 133-146.

27. RTSinfo [En ligne] « Les Suisses s'opposent massivement aux réseaux de soins » [mis à jour le 18 juin 2012, consulté le 27 mai 2019] https://www.rts.ch/info/suisse/4070154-les-suissess-opposent-massivement-aux-reseaux-de-soins.html

28. Etat de Vaud [En ligne] «Avant-Projet mis en consultation / Exposé des motifs et projet de loi sur les Régions de Santé (LRSa) abrogeant les lois sur l'Association vaudoise d'aide et de soins à domicile (LAVASAD), sur les Réseaux de soins (LRS) / modifiant la loi du 29 mai 1985 sur la Santé publique (LSP), la loi du 5 décembre 1978 sur la planification et le financement des établissements sanitaires (LPFES), la loi du 24 janvier 2006 décembre 1978 d'aide aux personnes recourant à l'action médico-sociale (LAPRAMS) / et répondant au postulat Claudine Wyssa et consorts « Analyse des coûts de la prise en charge des personnes dépendantes » [consulté le 27 mai 2019] https://www.vd.ch/fileadmin/user_upload/themes/sante/Avant-projet_de_loi_sur_ les_R\%C3\%A9gions_de_Sant $\%$ C3\%A9.pdf

29. Etat de Vaud [En ligne] « Retour de la consultation sur les Régions de Santé : orientations du Conseil d'Etat sur les suites à donner » [consulté le 27 mai 2019] https://www.bicweb.vd.ch/ frame.aspx?pPage $=/$ communique.aspx?pObjectID $=633920$

30. Réseau Santé Région Lausanne [En ligne] « Projet Anticipé des Soins » [consulté le 27 mai 2019] https://www.reseau-sante-region-lausanne.ch/projet-anticipe-des-soins-pas

31. CHUV [En ligne] « Chaire de soins palliatifs gériatriques » [consulté le 27 mai 2019] https://www.chuv.ch/fr/fiches/chaire-de-soins-palliatifs-geriatriques/

Artigo apresentado em: dezembro 2018 Artigo aprovado em: abril 2019 Conflito de interesses: o autor declara não haver conflito de interesses Financiamento: não 